\title{
La difficile territorialisation de la gestion de l'eau au Burkina Faso : une lecture au filtre de la théorie de la proximité
}

Challenging territorialization of water management in Burkina Faso: analysis through the framework of proximity

Bio Mohamadou Torou, Liza Debevec et Dapola Évariste Constant Da

\section{OpenEdition}

Journals

Édition électronique

URL : http://journals.openedition.org/developpementdurable/12046

DOI : 10.4000/developpementdurable.12046

ISSN : 1772-9971

Éditeur

Association DD\&T

\section{Référence électronique}

Bio Mohamadou Torou, Liza Debevec et Dapola Évariste Constant Da, «La difficile territorialisation de la gestion de l'eau au Burkina Faso : une lecture au filtre de la théorie de la proximité », Développement durable et territoires [En ligne], Vol. 9, $n^{\circ} 1$ | Mars 2018, mis en ligne le 30 mars 2018, consulté le 01 mai 2019. URL : http://journals.openedition.org/developpementdurable/12046 ; DOI : 10.4000/ developpementdurable.12046

Ce document a été généré automatiquement le 1 mai 2019.

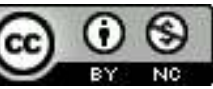

Développement Durable et Territoires est mis à disposition selon les termes de la licence Creative Commons Attribution - Pas d'Utilisation Commerciale 4.0 International. 


\title{
La difficile territorialisation de la gestion de l'eau au Burkina Faso : une lecture au filtre de la théorie de la proximité
}

\author{
Challenging territorialization of water management in Burkina Faso: analysis \\ through the framework of proximity
}

Bio Mohamadou Torou, Liza Debevec et Dapola Évariste Constant Da

Cette recherche a été financée en partie par le projet " Managing water and food systems in the Volta - Niger basins » avec un financement de la Commission européenne (CE) et un appui technique du Fonds international de développement agricole (FIDA).

1 À la suite de la conférence de Dublin en 1992, la gestion intégrée des ressources en eau (GIRE) s'est progressivement imposée comme étant la réponse principale aux problèmes du secteur de l'eau. Bien que sujet à une diversité d'interprétations ${ }^{1}$, le concept de GIRE s'est constitué comme étant un processus de gestion de l'eau fondé sur des principes d'intégration et de subsidiarité à l'échelle du bassin versant. Il s'agit de prendre en compte l'ensemble des interdépendances socio-spatiales dans le bassin (GWP, 2000) et d'amener les usagers à co-construire, avec les représentants locaux de l'État et les collectivités territoriales, des pratiques de gestion durable des ressources en eau.

L'adoption de l'échelle bassin versant et de la gestion multi-acteurs fait de la coordination des acteurs un élément central de la GIRE (Grigg, 2008; Gallego-Ayala, 2013). Cette coordination prend souvent la forme des plateformes multi-acteurs (PMA) très populaires dans le secteur de l'eau (Warner et al., 2008). L'enthousiasme généralisé pour ces PMA serait lié au fait qu'elles semblent apolitiques et qu'elles sont réputées reposer sur une démocratie à la base (Warner, 2006; Warner et Simpungwe, 2012). Cependant, le fonctionnement concret des PMA montre un écart entre les faits et l'idéal délibératif et d'implication des populations dans la décision collective qui sous-tendent leur création (Blondiaux et Sintomer, 2009). Il est souvent difficile de garantir une démocratie au sein 
de ces plateformes, car la représentativité (niveau d'inclusivité, d'équité et de liberté) en leur sein dépend aussi bien du contexte sociopolitique local (niveau élevé d'inégalités, hiérarchie sociale rigide) que de la volonté politique de l'État central d'impliquer réellement d'autres acteurs (Warner et Simpungwe, 2012 ; Mehta et al., 2014). Toutefois, la généralisation observée des PMA peut permettre la prise en compte de points de vue jusque-là ignorés dans le processus de décision (Blondiaux et Sintomer, 2009).

3 En lien avec les principes de la GIRE, des PMA dénommées Comités locaux de l'eau (CLE) ont été créées par les autorités burkinabés pour gérer l'eau à l'échelle des sous-bassins locaux. Le CLE est une entité décentralisée qui est supposée agir comme un forum de consultation au niveau local entre toutes les parties prenantes ayant un intérêt dans la gestion de l'eau à l'échelle d'un sous-bassin (Roncoli et al., 2009). L'objectif est d'ériger ces sous-bassins en nouveau territoire pour la gestion de l'eau, c'est-à-dire des espaces où la proximité géographique et les liens de confiance permettent des dynamiques de collaboration entre les acteurs (Barnèche-Miqueu et Lahaye, 2005). Cet article s'intéresse à la mise en œuvre de la GIRE, en mettant l'accent sur le rôle des CLE dans la construction et la gouvernance des territoires de l'eau au Burkina Faso.

Partant de la caractérisation de la territorialisation comme "la mise en place d'une organisation et d'une structuration nouvelles et spécifiques, matérielles et/ou idéelles, d'une portion d'espace par un groupe social» (Ghiotti, 2006:3), nous considérons la création des CLE comme un processus de construction de territoires de l'eau. Leur mise en place se fonde sur un double espoir que les CLE soient adaptés et modelés selon les spécificités et les enjeux locaux, et qu'ils contribuent à modifier, conforter, défaire ou reconstruire les territorialités existantes. Cependant, cette territorialisation est souvent peu aboutie comme bien d'autres dynamiques territoriales passées dans le domaine de l'eau au Burkina Faso. La raison est que les processus qui sont restés toujours descendants ne tiennent pas compte des dynamiques propres de la zone d'implantation (Venot et al., 2014); celles-ci étant considérées comme une simple toile de fond, un contexte secondaire (Zoungrana, 2010). Et pourtant, il faut considérer ce contexte comme un élément actif qui permet de garantir une appropriation des nouvelles structures, donc de leur durabilité (Zoungrana, 2010 ; Sally et al., 2011 ; Venot et al., 2014).

5 La création des CLE vise, en outre, à construire des coordinations d'acteurs capables de donner une cohérence à l'action publique locale (DGIRH, 2004) d'une part, et à minimiser les conflits d'usages, valoriser et protéger les ressources en eau (Sally et al., 2011 ; Venot et al., 2014) d'autre part. À ce titre, le CLE apparait comme un dispositif de gouvernance territoriale (Leloup et al., 2005) de l'eau au Burkina Faso. Dès lors, l'émergence des CLE devrait permettre l'établissement de relations fonctionnelles entre acteurs de l'eau dont les parcours, représentations, formats d'action, intérêts ou légitimités sont différents. Ceci suppose un processus permanent d'interaction, des jeux de négociation, de compromis et d'alliances entre les acteurs mobilisés en vue d'une convergence des représentations vis-à-vis de la ressource et de sa gestion. Ce jeu complexe relève de la mobilisation des relations de proximité (géographique et non géographique) dont l'objectif est, entre autres, de faciliter la coordination des groupes d'acteurs hétérogènes (Torre et Beuret, 2012). L'analyse de telles dynamiques d'action collective invite à examiner les propriétés du lien social. Les économistes de la proximité (Gilly et Torre, 2000 ; Pecqueur et Zimmerman, 2004), qui abordent la question des coordinations locales non marchandes, en proposent une grille d'analyse que nous mobilisons dans cet article. 
6 Si de nombreux travaux ont déjà souligné les difficultés d'action collective par les coordinations locales d'acteurs dans le cadre de la gestion de l'eau (Warner et al., 2008; Romagny et Riaux, 2009 ; Baron et Bonnassieux, 2013), ils n'ont pas mis l'accent sur le rôle des relations socio-spatiales dans le développement de la gouvernance locale de l'eau. Cette difficulté de coordination, aussi soulignée au niveau des CLE (BEGE et DGRE, 2008), est illustrée par une absence de décisions et d'actions collectives (Sally et al., 2011; Venot et al., 2014) sur leurs espaces de compétence; cette action collective qui transforme l'espace géographique en territoire (D'aquino, 2002). Nous faisons l'hypothèse que les localisations dans l'espace social et dans l'espace géographique des acteurs de l'eau sont déterminantes dans la construction des territoires de l'eau, et que l'application du cadre d'analyse de la proximité permet de comprendre ces dynamiques territoriales. Les éléments empiriques de l'article sont issus de deux cas d'étude de CLE au Burkina Faso : le Kou et Yitenga (Figure 1).

7 Après un bref aperçu du contexte de la gestion des ressources en eau et de la GIRE (section 1), nous présentons le cadre d'analyse de la proximité et décrivons en quoi ce cadre appliqué à nos deux CLE permet de renouveler le discours classique sur les difficultés de mise en œuvre de la GIRE (section 2). Nous montrons ensuite que, même si la mise en place des CLE fait naître une nouvelle proximité organisée basée sur le partage de quelques règles et instruments de gestion et l'émergence de préoccupations communes (section 3), les CLE étudiés n'ont pas réussi à construire une vision partagée d'un projet durable de territoire (section 4).

\section{L'évolution des modalités d'accès et d'usage de l'eau, et son impact sur la gestion locale de l'eau}

8 La lecture de la dynamique de développement des ressources en eau au Burkina Faso révèle deux grandes phases avant le lancement du processus de GIRE. La première phase, caractérisée par des politiques de l'eau centrées sur la réalisation d'infrastructures hydrauliques par l'État et ses partenaires, fait suite aux sécheresses des années 1970 et 1980 dans le Sahel. Dans les zones semi-arides, les petits réservoirs sont identifiés comme une option propice pour la mobilisation des ressources en eau, alors que dans les zones plus humides, l'État a privilégié les grands aménagements hydro-agricoles alimentés par captage de rivières permanentes ou de grands barrages. La gestion de ces infrastructures hydrauliques est assurée par les services déconcentrés de l'État. Cette gouvernance dite publique (Baron et Bonnassieux, 2013) vise surtout à asseoir la légitimité de l'État et ne laisse aucune place à l'initiative de l'utilisateur (Zougrana, 2010). La production de riz irrigué est imposée dans les aménagements en aval des barrages et, de plus, les grands aménagements sont exploités sous le mode du colonat. Les exploitants sont recrutés dans les régions surpeuplées du pays et réinstallés dans ces périmètres irrigués sous la supervision de l'administration publique. Ces migrations visent, selon l'État burkinabé, à limiter l'exode rural et, en même temps, à rééquilibrer les densités de population (Palé, 2012). L'irrigation dans les périmètres est dite formelle parce que planifiée et promue par l'État .

9 La seconde phase, au début des années 1990, marquée par les programmes d'ajustement structurel, voit le désengagement de l'État des différents secteurs, dont celui de l'eau. Les usagers, désormais organisés en coopératives de production agricole et/ou en comités de 
gestion de barrage (Zougrana, 2010), prennent en charge la gestion de l'eau alors qualifiée de gouvernance communautaire (Baron et Bonnassieux, 2013). En l'absence de l'autorité publique, cette phase a donné lieu au développement d'une irrigation privée de contresaison, en amont des réservoirs et le long des rivières et canaux d'irrigation. Cette pratique, bien que tolérée, n'est pas autorisée et est dénommée « irrigation informelle ». En général consacrée au maraîchage, elle est plus rentable que la culture du riz et occupe un espace beaucoup plus grand que la zone aménagée (De Fraiture et al., 2014). Il s'ensuit une multiplication des conflits d'usages de l'eau, conséquences des pressions exercées sur la ressource et de la perte de droits exclusifs d'accès entre-temps gardés par les exploitants des périmètres irrigués.

10 La recherche de nouveaux compromis est devenue alors une priorité politique et l'interprétation du système hydraulique comme intégré à un territoire plus vaste, une nécessité. Ceci s'est concrétisé par l'adoption des conventions internationales et des plans d'action au niveau national selon les principes de la GIRE (DGRE, 2006).

11 La réforme de la gouvernance de l'eau au Burkina Faso a abouti à l'adoption de la loi sur la gestion de l'eau de $2001^{2}$, dont la mise en œuvre s'est traduite dans un plan d'action (PAGIRE) adopté par décret en 2003. La mise en œuvre du PAGIRE (2003-2015) a permis la réforme du cadre institutionnel de gestion des ressources en eau avec la mise en place de structures de gestion et de cadres de concertation à différents niveaux, de l'échelle locale à l'échelle des bassins versants nationaux. La figure 1 présente les quatre bassins nationaux qui sont, en fait, des sous-bassins de fleuves africains dans les limites territoriales du pays : la Comoé, le Niger, le Nakambé (Volta blanche) et le Mouhoun (Volta noire). Dans une optique d'opérationnalisation et en tenant compte des contraintes administratives et biophysiques, le territoire est divisé en cinq espaces de gestion qui ne recoupent que partiellement les limites hydrographiques. C'est à l'échelle de ceux-ci que les Agences de l'eau sont créées.

Les agences de l'eau sont des Groupements d'intérêt public (GIP) convenus entre l'État et les collectivités territoriales ayant compétence sur l'ensemble d'un bassin défini comme espace de gestion des ressources en eau. Elles coordonnent les actions relatives à la planification et la gestion par la concertation afin de préparer et de mettre en œuvre, dans les conditions optimales de rationalité, les orientations et les décisions prises dans le domaine de l'eau. Chaque agence est dotée d'un Comité de bassin (organe délibératif), d'un Comité interservices de l'eau (structure technique), d'un conseil d'administration (organe exécutif) et des Comités locaux de l'eau (CLE) (instances locales). Le processus de création des CLE est placé sous le contrôle des agences de l'eau, même si les différents bailleurs s'efforcent de marquer le processus de leur empreinte. Un CLE est composé de représentants de l'État (administratifs et techniques), des usagers de l'eau, de la société civile (ONG locales intervenant dans le domaine de l'eau) et des collectivités locales (communes). Ils n'ont pas de pouvoir décisionnaire et doivent rendre compte à l'agence compétente sur leur espace géographique (DGRE, 2010). Ils sont responsables de l'élaboration des schémas d'aménagement et de gestion de l'eau (SAGE) de leur zone de compétence.

13 Un nouveau programme national GIRE-PNGIRE (2016-2030) est lancé en mai 2016 avec pour objectif stratégique de contribuer durablement à la satisfaction des besoins en eau douce des usagers et des écosystèmes aquatiques. En plus du renforcement du cadre institutionnel et législatif auquel le PAGIRE a été consacré, le PNGIRE vise l'amélioration 
de l'autofinancement du secteur et la protection des ressources en eau, et prend en compte le nouveau contexte national dont, notamment, le processus de décentralisation.

Figure 1. Bassins versants nationaux et situation des sites d'étude

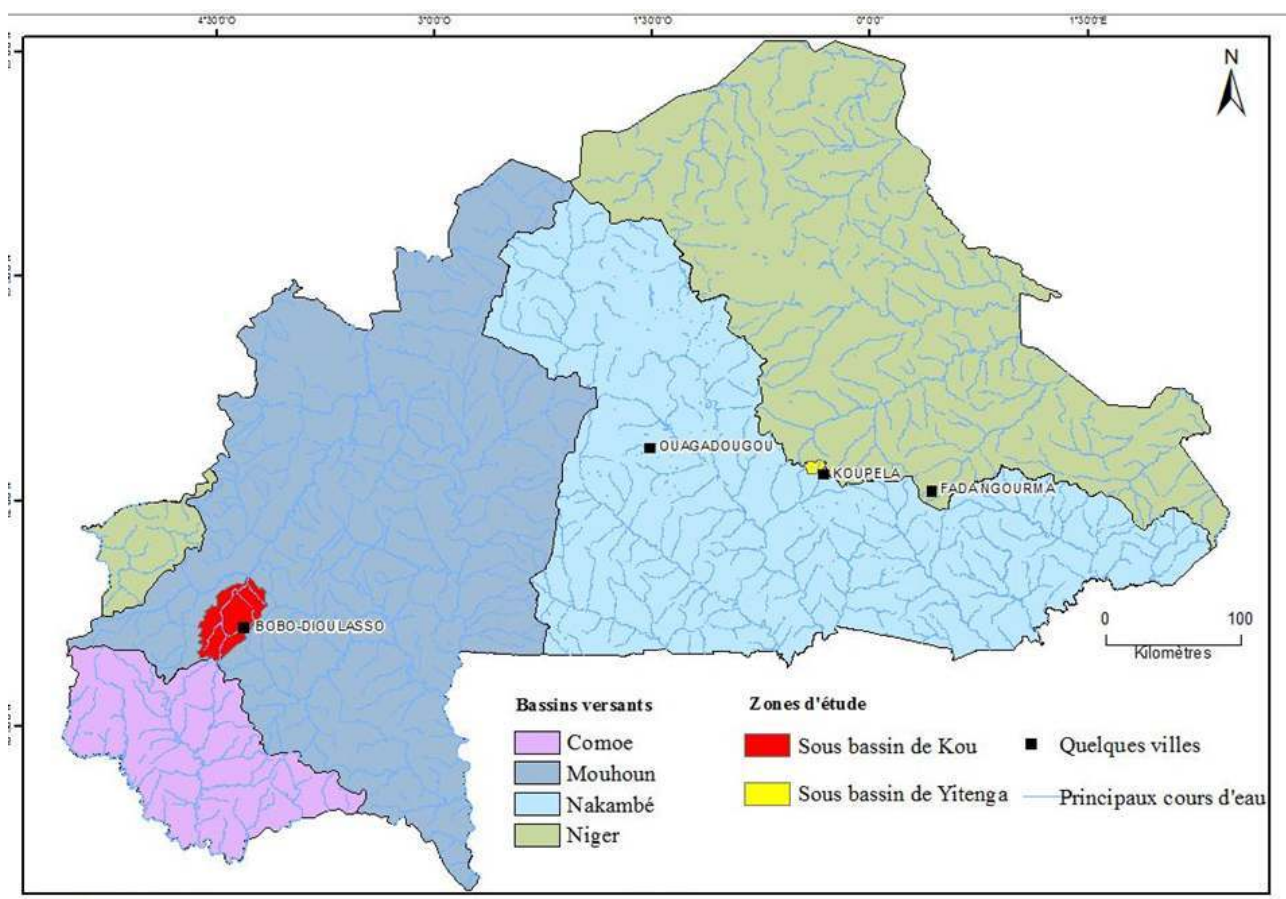

Source : BNDT, 2012, Projet VREO - mars 2016

\section{L'approche de la proximité et le processus de construction territoriale : le cadre d'analyse}

\subsection{Approche de la proximité et son intérêt dans l'étude des CLE}

Issue de l'économie du développement territorial (RERU, 1993; Rallet et Torre, 1995), l'approche de la proximité pose la question de l'influence de la localisation des acteurs dans l'espace géographique et dans l'espace social sur les processus de coordination. Elle met l'accent sur l'action et les stratégies des acteurs considérés dans leur environnement géographique et relationnel (Pecqueur et Zimmermann, 2004). La littérature sur les analyses de proximité définit une pluralité de formes de proximités, institutionnelle, organisationnelle, cognitive, sociale et géographique (voir Talbot, 2010) et reflète une pluralité de courants théoriques (interactionnisme, institutionnalisme, évolutionnisme, théorie de la régulation, théorie des organisations, etc.). Mais tous proposent une grille d'analyse qui s'articule autour de dimensions géographiques et non géographiques (voir Carrincazeaux et al., 2008). Parmi la multitude de déclinaisons des proximités, deux courants fondamentaux (interactionniste et institutionnaliste), qui font respectivement appel à deux types de proximités (géographique et organisée) ou trois (géographique, institutionnelle et organisationnelle), ont émergé, notamment en ce qui concerne la lecture des dynamiques territoriales.

15 L'acception de la proximité géographique fait l'objet d'un large consensus. Elle traduit la distance kilométrique entre deux entités, pondérée par les délais et les coûts de son 
franchissement. Elle renseigne sur la position relative des entités dans un espace plan déterminé, "près de » ou "loin de». On peut donc parler de proximité géographique entre des acteurs humains, entre des lieux ou des objets techniques, ou encore entre des acteurs humains d'une part et des lieux ou des objets techniques d'autre part (Torre, 2009). Nous nous intéressons ici à la proximité géographique en rapport avec les usages de l'eau.

La dimension non géographique de la proximité fait moins consensus dans la littérature. Elle regroupe les différentes manières qu'ont les acteurs d'être proches en dehors de la relation géographique (Torre, 2009). Derrière cette définition se cachent plusieurs types de proximités non géographiques bien distinctes. La distinction porte, de façon globale, sur la nature du lien social interindividuel ou collectif. La séparation entre proximités institutionnelle et organisationnelle prônée par le courant institutionnaliste vise la prise en compte des repères collectifs des acteurs et donc à questionner la coordination suivant deux dimensions, cognitive et politique ; la dernière n'étant pas prise en compte par les interactionnistes (Talbot, 2010). Mais le plus important pour nous, ce sont les modalités et la finalité de l'action des acteurs, au-delà de leur statut d'individus isolés ou d'organisations. C'est ce qui justifie notre adhésion au paradigme interactionniste qui pose que les dynamiques territoriales peuvent être expliquées uniquement à travers des interactions interindividuelles. En effet, notre étude des CLE prend pour unité de base l'interaction entre les acteurs de l'eau. C'est pourquoi, dans le cadre de notre analyse, nous retenons un seul type de proximité non géographique, la proximité organisée avec ses deux logiques : la logique d'appartenance et la logique de similitude. L'appartenance à une organisation se traduit par l'existence d'interactions entre ses membres. De ce point de vue, sont proches des acteurs entre lesquels s'établissent des interactions facilitées par les règles ou les routines de comportement (explicites ou tacites) qu'ils observent. La logique de similitude renvoie plutôt à l'espace de représentation des individus. Selon cette dernière acception, sont désignés proches des agents qui se ressemblent et qui partagent le même espace de valeurs, de règles, de modèles de pensées et d'actions, ce qui facilite leur capacité à interagir.

Bien qu'initialement focalisée sur les relations productives, l'approche de la proximité a progressivement porté sur des domaines de plus en plus larges comme le transport, l'emploi, le développement durable et la politique de la ville (Gomez et al., 2011). Mais, c'est l'application des catégories de la proximité au domaine de l'environnement qui a induit des mutations fondamentales au cœur même du paradigme de recherche (Torre et Zuindeau, 2009). La prise en compte d'acteurs extérieurs à la sphère productive (ménages, associations, collectivités territoriales), la mise en exergue des dimensions "négatives " de la proximité géographique et l'introduction de la proximité géographique par rapport aux objets (Torre, 2009) ont conduit à changer le sens des proximités mobilisées ainsi que leur importance relative dans l'explication des coordinations ou absences de coordination observées. Ces apports, en s'intéressant aux modes de coordination entre acteurs pour l'action collective et aux explications des origines et des causes des conflits, introduisent une dissonance au cœur de l'action, permettant ainsi de rompre avec la vision coopérative et résiliaire des processus de coordination ou de gouvernance locale (Torre et Zuindeau, 2009).

18 La mobilisation de l'approche de la proximité pour l'étude des CLE entre dans ce cadre. En effet, l'approche se prête bien à l'étude de la gestion de l'eau au niveau local dans sa dimension spatiale, essentielle pour comprendre les enjeux de pollution et de partage, 
dans la délimitation des périmètres d'action publique et dans les modalités de structuration du lien social autour de la ressource. Les acteurs du CLE sont tenus par des relations de proximité géographique. Ils partagent le même espace hydraulique et sont supposés aisément se concerter. Et ces acteurs interagissent et entretiennent des relations sociales soit parce qu'ils appartiennent aux mêmes associations d'usagers (réseaux), soit parce qu'ils ont des objectifs communs (similitude), l'approvisionnement en eau potable par exemple. Ils sont ainsi liés par des relations de proximité organisée dans sa double dimension d'appartenance et de similitude. Puisque le CLE est supposé permettre une agrégation des points de vue pour définir des enjeux partagés et des règles de gestion construites collectivement, sa création et son fonctionnement relèvent de l'activation de relations de proximité, qu'elle soit géographique ou non géographique. Mais comment s'articulent ces deux catégories de proximités?

\subsection{Articulation des proximités, condition de construction de territoires de l'eau}

19 Proximité géographique et proximité organisée ne sont pas totalement indépendantes ni séparables. Elles sont corrélées et sont à la fois des conditions et des résultats des relations en général (Talbot, 2010; Torre et Beuret, 2012). L'activation de la proximité géographique facilite les interactions entre personnes et, par là même, l'échange de connaissances et l'augmentation de la confiance (Torre, 2009). Autrement dit, la proximité géographique peut favoriser la construction de proximités organisées, donc la coordination et la co-construction du résultat. Mais elle peut aussi générer des conflits de voisinage (pollution) ou d'usage de l'espace (Torre et Zuindeau, 2009; Torre et Beuret, 2012) qui mettent fin à un réseau collaboratif naissant : les proximités se détruisent. À l'opposé, la mobilisation des ressources de la proximité organisée par exemple, au travers de dispositifs locaux associant usagers, collectivités locales et services de l'État autour de la gestion et de la protection de la ressource en eau, peut permettre de réguler les conflits, qu'il s'agisse de les prévenir ou de les modérer par l'apaisement des tensions entre usagers de l'espace. Dans ce cas, les proximités se compensent. En outre, une trop grande proximité organisée peut aussi conduire à une ségrégation sociale et un repli sur soi. Enfin, proximité géographique et proximité organisée peuvent s'ignorer. Et c'est à ce moment qu'il devient nécessaire que les acteurs aient un projet stratégique pour activer ce potentiel de proximités, comme la création du CLE pour une gestion collective et apaisée de l'eau.

Le jeu des proximités organisées et géographiques constitue ainsi le ferment de la création et de la dynamique des territoires, et est à la base des processus de gouvernance territoriale (Torre et Beuret, 2012). Ce faisant, elles contribuent autant à unifier les territoires qu'à les fragmenter ou les recomposer : les proximités évoluent au gré des nouveaux enjeux et des échelles de gestion pertinentes au vu de ces enjeux. C'est précisément le parti pris ici, puisque nous tenterons de démontrer comment la proximité géographique autour de l'eau a déterminé, dans un premier temps, des relations tendues entre certains acteurs et, ensuite, une réorganisation de la gestion de l'eau à travers le CLE (proximité organisée), même si celle-ci n'a pas encore abouti à une totale synergie d'action. 


\section{Les proximités comme outils de construction territoriale : les cas des CLE Kou et Yitenga}

\subsection{Contexte et méthodologie}

Les deux cas décrits dans cet article ont été étudiés dans le cadre du projet de recherchedéveloppement $\mathrm{CPWF} / \mathrm{V} 4^{3}$. Dans chacun se posent des problèmes de partage de l'eau liés aux configurations d'espace, et aux questions de droits d'accès et d'usage de la ressource. Les sous-bassins de Yitenga et du Kou ont été choisis parce qu'ils illustrent les défis pratiques de la gestion de l'eau dans le cadre de la GIRE au niveau local au Burkina Faso. Le premier est un sous-bassin où les enjeux de l'eau sont concentrés autour d'un réservoir doté d'un petit périmètre en aval ( $48 \mathrm{ha}$ ), d'une histoire plus récente (fin des années 1980), alors que le second est une rivière captée depuis 1970 pour alimenter un grand périmètre rizicole $(1260 \mathrm{ha})$ dont l'exploitation s'accompagne d'une histoire de peuplement du bassin. Pourtant, ces sites ont aussi en commun les problématiques de gestion de l'eau, à savoir le développement de l'irrigation informelle à côté de l'irrigation formelle d'une part, et la primauté de l'eau potable sur les usages agricoles d'autre part. La concurrence territoriale autour de l'eau se pose sur les deux sites avec des similitudes, mais aussi des différences. Dans le premier cas, ce sont deux communes qui se disputent le contrôle de la ressource par acteurs interposés et, dans le second cas, c'est une confrontation de droits de propriété et d'usage (foncier coutumier/droit moderne) de l'eau sur fond d'opposition autochtones/migrants.

Les données ont été collectées à partir de 75 entretiens semi-directifs auprès des usagers, des services techniques déconcentrés, des collectivités territoriales et des ONG intervenant dans le domaine de l'eau. Les données collectées en deux phases successives (mars-juin 2011 et juin-septembre 2012) sont complétées par un suivi des CLE jusqu'en avril 2015 dans le cadre d'une thèse. L'étude avait un double objectif : (1) identifier les activités et les problèmes liés à l'eau dans l'espace de compétence de ces deux CLE; et (2) cerner les réseaux d'acteurs mobilisés et les relations qu'ils entretiennent dans le cadre de la gestion de l'eau. Pour y arriver, nous avons utilisé le bureau du CLE ${ }^{4}$ comme point d'entrée, car le CLE est la structure centrale pour la gestion de l'eau dans sa zone de compétence. Chaque membre du bureau interrogé génère trois noms d'acteurs avec lesquels il collabore et qualifie la nature de la collaboration qu'il a avec ces individus. Les acteurs générés sont ensuite interrogés pour confirmer l'effectivité de la relation.

Les résultats de ce travail permettent de caractériser les relations dans les deux zones d'étude (en termes de densité, de nature et des raisons qui motivent la relation et les catégories d'acteurs impliqués). Ils révèlent des régularités qui transcendent les spécificités locales en matière de processus de coordination et d'action collective au niveau local.

Un important travail de documentation a aussi été réalisé à partir de rapports professionnels, et de nombreux documents fournis par les organisations d'usagers de l'eau, des différentes administrations et services concernés par la gestion de l'eau. 


\subsection{Les CLE : la construction des territoires par les proximités}

\subsubsection{Des espaces d'interdépendances et d'interaction face à face autour de l'eau} hydraulique de la rivière du Kou, ses affluents et les sources de Nasso. C'est le sous-bassin hydrographique de la rivière Kou, le long duquel se concentrent les enjeux de l'eau. Celui du CLE de Yitenga prend en compte, en plus du bassin d'alimentation du barrage de Yitenga, la ville de Koupéla située à 3 kilomètres en aval. C'est le bassin d'usages de l'eau du réservoir.

La construction des territoires de l'eau au Burkina Faso a commencé par la délimitation des zones de compétence des CLE. Celle-ci répond à un double souci de favoriser les interactions entre acteurs, et de cerner les interdépendances naturelles et techniques autour de la ressource en eau. C'est ce qui justifie que les premières expériences de CLE mettaient l'accent sur l'organisation des acteurs autour des points d'eau, perçus alors comme de "puissants facteurs de mobilisation, d'adhésion et de participation effective des populations" (BEGE et DGRE, 2008, p.54) et qui constituent des lieux où se cristallisent les interactions entre différents acteurs (Venot et al., 2014). Actuellement et en règle générale, les parties prenantes au CLE doivent être localisées dans un sous-bassin local dont la superficie ne devrait pas excéder $3000 \mathrm{~km}^{2}$ (DGRE, 2010). Le partage du même espace hydraulique serait un atout pour la concertation.

Dans les deux territoires, deux types d'interdépendances (qualitatives et quantitatives) sont identifiés.

Figure 2. Schéma des interdépendances autour de l'eau dans le Kou

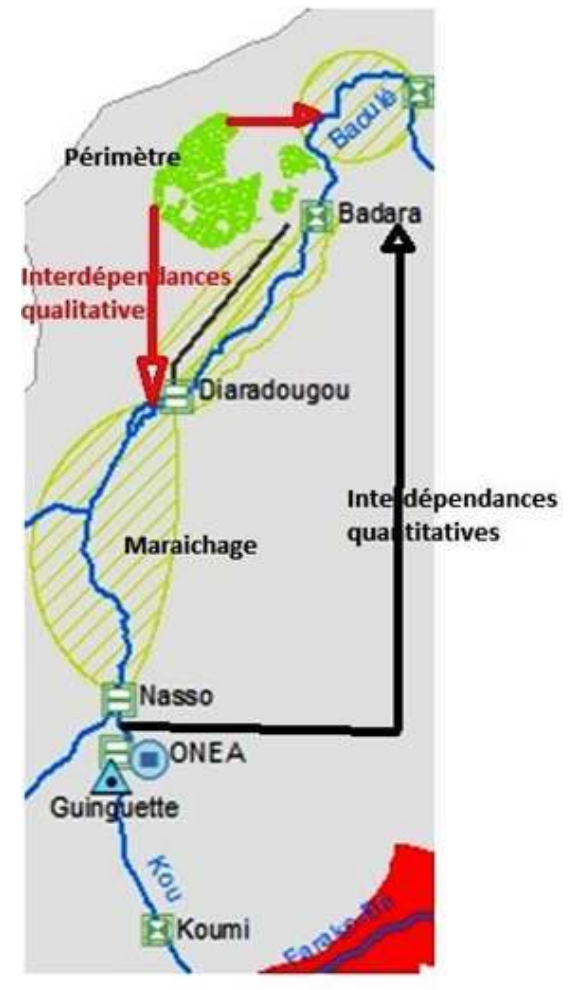

Sources : Auteur à partir d'un morceau de Wellens et Compaoré, 2005 
La figure 2 laisse voir une succession de zones de consommation d'eau dans le Kou et le lien amont-aval qui les caractérise. D'un point de vue quantitatif, la gestion de l'eau dans le bassin du Kou en période de basses eaux consiste en la satisfaction, d'une part des besoins en eau du périmètre rizicole (en vert) et, d'autre part, des besoins en eau des espaces maraîchers le long du canal d'amenée et du cours d'eau (en vert rainuré) situés en amont de la prise d'eau, au niveau du village de Diaradougou. Cette gestion se fait dans un contexte singulier où le débit de base du Kou est lié principalement aux apports des sources de Nasso après prélèvement de l'eau nécessaire à l'alimentation en eau potable de la ville de Bobo-Dioulasso (en rouge). Du point de vue environnemental, des risques de pollution des eaux du fleuve par les eaux usées domestiques et industrielles de la ville de Bobo-Dioulasso sont évoqués. Les cultures sur les berges causent l'ensablement du fleuve et surtout du canal dont le nettoyage provoque des inondations dans les villages en amont de la prise d'eau et sont parfois source de conflits, comme ce fut le cas à Diaradougou en $2010^{5}$.

29 Cette configuration spatiale du bassin est le résultat des politiques de développement rural dans le bassin. Le périmètre irrigué de la vallée du Kou est exploité par des migrants mossi installés par l'État, qui en assurait la gestion jusque dans les années 1990 (Diallo et al., 2006). Les autochtones (Bobo), qui n'avaient pas accès à l'eau, ont profité de la fin de la tutelle administrative pour développer les activités d'irrigation en amont, réduisant ainsi la quantité d'eau qui parvient au périmètre (Wellens et Compaoré, 2005). La pénurie est aggravée par le tarissement de cinq des neuf sources qui soutiennent l'étiage du fleuve Kou. Depuis, le partage de l'eau est devenu houleux entre irrigants formels et informels, chaque groupe se prévalant d'un droit d'accès et d'usage de l'eau. Les migrants ont la concession officielle de l'État et misent sur la légalité portée par la domanialité de l'eau. Les irrigants informels se basent eux sur le droit foncier coutumier pour réclamer leur plein accès à l'eau. 
Figure 3. Schéma des interdépendances autour du réservoir de Yitenga

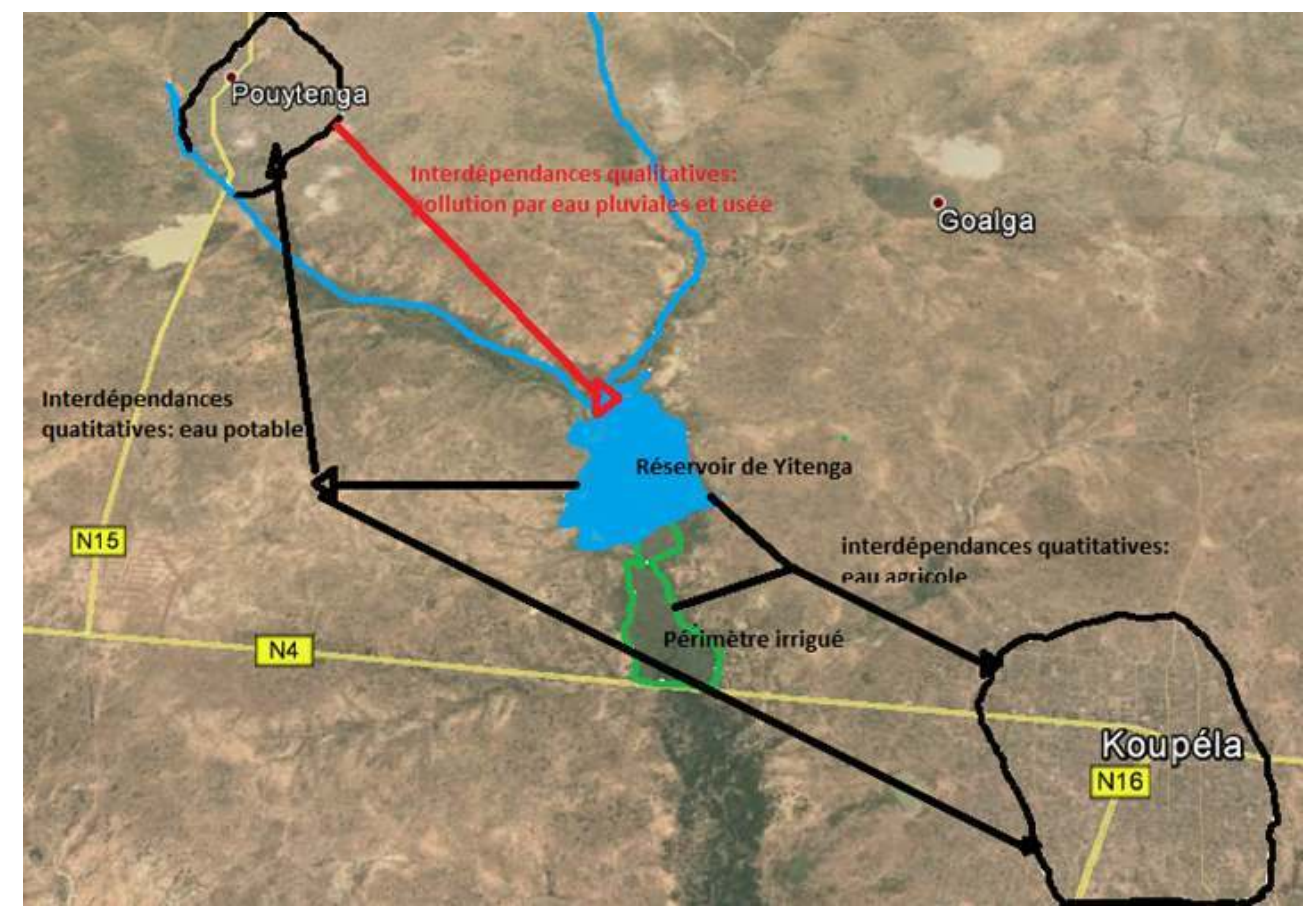

Source : Auteur à partir d'images Google Earth, 2014

$\mathrm{Au}$ niveau de Yitenga, les interdépendances sont à la fois naturelles et techniques. La figure 3 montre que les ruisseaux qui alimentent le réservoir ceinturent la ville de Pouytenga. Les eaux usées et de ruissellement, et les déchets d'abattage de bétail de la ville sont drainés dans le réservoir et causent une pollution physique et bactériologique des eaux. Les responsables de la société de fourniture d'eau potable (Office national de l'eau et de l'assainissement-ONEA) estiment la turbidité de l'eau du barrage en moyenne à 350 NTU et pouvant atteindre 6000 NTU lors des premières pluies alors que la norme acceptable est de $50 \mathrm{NTU}^{6}$.

31 L'interdépendance quantitative entre les deux villes de Koupéla et de Pouytenga, distantes seulement de 10 kilomètres, est induite par l'implantation de la station de pompage de l'ONEA. La société, déjà installée dans la ville de Koupéla, a profité de cette proximité pour étendre son réseau d'adduction à la ville de Pouytenga, en pleine croissance. Les irrigants (formels et informels) qui exploitent le réservoir viennent de la ville de Koupéla et doivent désormais partager l'eau avec les habitants de la ville voisine par l'entremise de l'ONEA. Le réservoir de Yitenga, au regard de nombreuses sollicitations dont il est l'objet, n'est plus à même de répondre à sa vocation initiale qui était d'assurer l'irrigation de la plaine en aval. Cette vocation a été modifiée en donnant priorité à l'eau potable. De fait, les usagers agricoles de l'eau vivent la présence de la station de pompage de l'ONEA et la proximité de la ville de Pouytenga comme un problème.

Malgré des configurations spatiales différentes (linéaire dans le Kou et ponctuelle à Yitenga), on note, dans les deux territoires en construction, que les interdépendances de proximité sont durement ressenties par tous les acteurs, surtout en situation de pénurie. La question des usages de l'eau cristallise alors les problèmes d'ajustement et d'adaptation de ces divers usagers. Pendant longtemps ils ont semblé s'ignorer et chaque espace de consommation est géré de façon autonome comme si ce n'était pas la même 
ressource qui était exploitée. Mais au fur et à mesure que les impacts des actions des uns sur les autres s'accentuent, les tensions s'élèvent et parfois aboutissent à des conflits. Au niveau du Kou, depuis les années 1970, la gestion de l'eau prenait seulement en compte le périmètre irrigué d'un côté, et l'ONEA de l'autre. Les irrigants informels ne sont pas associés et sont régulièrement délogés de leurs jardins par les services forestiers et la gendarmerie pour occupation illégale de l'espace public. Mais la tournure socioidentitaire que prenaient les tensions ont obligé les autorités administratives à appliquer des mesures allant dans le sens d'une gestion concertée et apaisée de l'eau entre les différents acteurs, contraints au compromis et plus généralement à la coordination. Tous agriculteurs (les autochtones et les migrants installés depuis 1976), il leur est difficile de se relocaliser pour échapper aux contraintes de proximité. Les négociations ont abouti à la création du Comité provisoire de gestion du bassin du Kou (CGBK). Des démarches similaires avaient été entreprises dans le bassin de Yitenga, mais elles n'ont pas abouti car les agriculteurs voient l'ONEA comme un imposteur. En effet, plusieurs actes posés concernant le partage de l'eau ont tendu l'atmosphère entre les irrigants et l'ONEA. Plusieurs fois en situation de pénurie, l'ONEA a ordonné la fermeture des vannes d'irrigation pour maintenir le niveau d'eau nécessaire au pompage. Les irrigants, quant à eux, vont ouvrir les vannes de nuit, et parfois saboter les installations de l'ONEA. Cette situation a poussé le service régional d'hydraulique à engager des concertations d'abord entre structures administratives, puis ouvertes aux acteurs usagers que sont l'ONEA et les irrigants.

33 Au total, on peut retenir que si les effets de contiguïté et de voisinage ont provoqué, dans un premier temps, des tensions dans le partage de l'eau, ils ont aussi été déterminants dans la mise en place d'une dynamique de concertation entre des usagers de l'eau qui sont voisins et subissent les conséquences de leurs actions respectives. Ces processus de concertation ayant conduit à la création du CGBK, ancêtre du CLE-Kou, et les négociations entreprises au niveau de Yitenga reposent sur des proximités organisées préexistantes, mais dont les potentiels vont être mobilisés au service de la gestion concertée de l'eau. Il s'agit de relations d'appartenance, car les participants se rattachent à des groupes déjà constitués (union de producteurs, associations) ou plus informels, mais également de relations de similitude, faites de références communes à des visions ou pratiques (irrigation formelle, informelle ou primauté de l'eau potable). La mobilisation des opportunités offertes par ces potentiels va-t-elle contribuer à construire les relations de coopération effective autour de l'eau?

\subsubsection{Le comité local de l'eau comme base d'une proximité organisée}

La mise en évidence des interdépendances spatiales autour de l'eau et des problèmes de gestion qu'elles engendrent pour les différents acteurs (usagers et services techniques) a favorisé les interactions; celles-ci ont permis l'établissement des relations entre des acteurs situés et, de ce fait, ont tenté d'instaurer les bases d'une nouvelle proximité entre les usagers de l'eau liés par une proximité géographique. Dans ce contexte, le CLE apparait comme un dispositif permettant de traiter les problèmes de partage de l'eau par le rapprochement des usagers concurrents, la construction de références communes et, pour finir, l'adhésion à un projet commun de valorisation et de protection de la ressource. Conformément à cette vision de gestion territoriale, le compromis au sein des CLE est censé s'exprimer à travers sa composition tripartite, la formalisation d'un projet commun

${ }^{7}$ et la production de règles partagées pour atteindre l'objectif commun de gestion 
concertée de l'eau. La création des CLE consacrerait ainsi une modalité nouvelle d'action locale dans le domaine des ressources en eau.

De nos enquêtes, il ressort que ce principe de base est respecté. Les arrêtés de création des CLE dans leur ensemble indiquent que ces derniers doivent être des cadres communs de production de règles issues d'un compromis entre les représentants de l'administration, des collectivités et des usagers pour résoudre de façon concertée les problèmes de gestion de l'eau. Ce compromis commence par la représentation de toutes les catégories d'acteurs au sein du CLE comme l'indique le tableau 1. Ce tableau décrit la composition des assemblées générales des CLE étudiés. La forte représentation des usagers provient de leur diversité et de la difficulté à désigner leurs représentants.

Tableau 1. Composition des assemblées générales des CLE du Kou et de Yitenga

\begin{tabular}{|l|c|c|}
\hline Collèges d'acteurs & CLE Kou & CLE Yitenga \\
\hline Administration & 7 & 11 \\
\hline Collectivités & 4 & 4 \\
\hline Usagers et Société civile & 30 & 18 \\
\hline Total & 41 & 33 \\
\hline
\end{tabular}

36 Aussi, les deux CLE étudiés ont établi des règles de partage et de protection de l'eau concernant respectivement :

- les pratiques de prélèvement d'irrigation consistant à réduire les surfaces à irriguer en période d'étiage (la surface à irriguer sur la plaine de Yitenga est réduite de moitié, abandon de la riziculture de contre-saison dans la plaine de Bama dans le Kou);

- la délimitation des espaces de protection des berges, des cours et plans d'eau (reboisement des berges du réservoir de Yitenga et cours principal du Kou, protection des sources de Nasso - zone de pompage d'eau de l'ONEA à Bobo) ;

- les procédures de prises de décision orientées par un principe de concertation et encadrées par deux textes fondamentaux que sont les statuts et le règlement intérieur du CLE.

37 Toutefois, ces règles relèvent du principe d'agir ensemble. Comme nous le développons par la suite, seules les modalités d'application nous édifieront sur le suivi et l'impact de ces règles sur la gestion de l'eau.

Dans tous les cas, les CLE étudiés ont fourni un cadre de dialogue et de concertation pour les acteurs en tension, animé par des réunions périodiques et extraordinaires en cas de problèmes urgents à traiter. Pour les uns, le CLE permettait enfin le rétablissement de l'équité et de la justice sociale dans le partage de l'eau, et pour les autres, il permettrait de régler définitivement une situation conflictuelle par la négociation et la concertation. $\mathrm{Au}$ niveau social, le gain est certain car, grâce aux arènes de discussion, le partage de l'eau est moins conflictuel et les acteurs de l'eau ont désormais la plupart du temps un référent. L'activation de la proximité organisée permet, à travers la formalisation de cette arène de discussion (le CLE), le développement d'un sentiment d'appartenance à une organisation (logique d'appartenance). La proximité géographique est activée par la mobilisation de la logique d'appartenance de la proximité organisée.

Eu égard à ce qui précède, il convient de dire que le CLE est une organisation territoriale caractérisée par la proximité géographique entre les acteurs, sa capacité à rassembler des 
groupes sociaux différents et de permettre le débat entre eux. Le fait de créer localement une telle structure où les acteurs puissent exprimer leurs perceptions, enrichir leurs savoirs et peser sur le processus est une autre étape de la construction d'un "nouveau » territoire, l'appropriation territoriale selon d'Aquino (2002). Pour autant, le sentiment d'appartenir à une communauté de gestion de l'eau à l'échelle du bassin n'existe pas encore, car beaucoup estiment que les intérêts de tous ne sont pas pris en compte.

\section{Les CLE à l'épreuve de la gestion de l'eau}

Bien que la création du CLE accroisse la convergence des acteurs pour une gestion concertée et durable de l'eau, ce dialogue naissant n'a pas concouru à inventer une responsabilité collective qui transcende les représentations et les intérêts territoriaux, identitaires ou socioprofessionnels. La gestion bute d'abord sur des problèmes inhérents aux moyens imaginés pour le partage et la protection de la ressource, à la mise en cohérence des intérêts privés et à la reconstitution de réseau d'acteurs selon de nouvelles affinités.

\subsection{Les limites inhérentes à la mobilisation des potentialités de la proximité géographique}

41 La première limite du CLE réside dans la mise en pratique des mesures de protection de l'eau (la délimitation d'une zone de marnage) et de la gestion volumétrique de l'eau (réduction des espaces à irriguer). Ces mesures semblent renforcer les priorités déjà établies, de sorte que les acteurs en situation défavorable par rapport aux règles et pratiques communes ont recours au conflit afin de faire valoir leurs intérêts. La question des droits d'accès et d'usage de l'eau est au centre de tous les enjeux. Les politiques publiques de l'eau définissent des priorités quant aux usages et à l'affectation des ressources en eau. Le code de l'eau accorde la priorité à l'approvisionnement en eau potable (AEP). Pour les autres usages, les priorités sont définies en tenant compte des spécificités locales.

Au niveau de Yitenga, c'est le changement d'affectation des ressources en eau qui fait problème. Les irrigants dénoncent la limitation des prélèvements agricoles en faveur de l'ONEA, résultat de priorité donnée à l'eau potable. Pour eux, le réservoir et l'aménagement sont leur propriété, parce que l'aménagement hydro-agricole est réalisé sur leurs terres et que l'État leur en a concédé l'exploitation et la gestion contre le paiement de redevances. Ils vivent donc ces mesures comme un diktat et une usurpation de leurs prérogatives par cette société en connivence avec les maires de Koupèla et de Pouytenga. Pour ces irrigants, "le maire de Koupèla les a trahis ${ }^{8}$ " car ils s'attendaient à ce qu'il les défende face à l'ONEA. Aussi, ils estiment que la réduction des prélèvements, contraire à leurs intérêts de production, n'est pas la meilleure solution, car l'ONEA aurait pu investir pour augmenter la capacité du réservoir. Une situation semblable s'observe dans le Kou où les maraîchers et fruitiers hors périmètre dénoncent les mesures de protection des berges. Elles leur rappellent le temps de la tutelle administrative du périmètre quand les irrigants informels ont été exclus. Pour le président de l'union des maraîchers, « le CLE ne leur prête pas une réelle attention ». 
La logique de similitude nous conduit à analyser la seconde limite du CLE, à savoir la persistance ou même le renforcement des clivages qui séparent les différents acteurs de l'eau. Au sein du CLE, les groupes d'acteurs qui partagent les mêmes objectifs ont tendance à écarter les autres, et à se constituer en groupes homogènes et isolés, à la fois $\mathrm{du}$ point de vue social et spatial. Le partage de l'objectif «approvisionnement en eau potable » a favorisé la coopération entre l'ONEA et les mairies de Koupéla et de Pouytenga qui en ont la prérogative. Ainsi décident-ils du partage de l'eau et mettent-ils souvent les agriculteurs devant le fait accompli. Les agriculteurs ont vu plus d'une fois leurs cultures desséchées faute d'eau. Un front d'opposition s'est développé entre tous les autres usagers agricoles contre l'ONEA et les responsables communaux, en guise de riposte fondée sur l'appartenance à la commune Koupéla, et qui s'est muée en rivalité territoriale contre celle de Pouytenga. «Le CLE appartient au département de Koupéla et les acteurs de Pouytenga ne peuvent prétendre en assumer la direction; ils ne sont que des pollueurs", s'exclamait la trésorière du bureau du CLE lors de nos entretiens. Face à cette hostilité grandissante, les responsables de la société envisagent une relocalisation de la station de pompage au niveau d'un réservoir plus grand et moins pollué. Au niveau du Kou, c'est plutôt l'objectif de production rizicole qui fédère l'administration et ses services 
techniques autour des irrigants de la plaine de Bama, au détriment des irrigants informels (maraîchers) présentés régulièrement comme la cause de l'insuffisance de l'eau. Cette opposition entre catégories d'irrigants prend parfois des relents de conflit identitaire entre les maraîchers autochtones du milieu et les riziculteurs de la plaine, colons agricoles. Ainsi, les catégories d'usagers s'affinent selon des représentations mues par des enjeux territoriaux et/ou socioprofessionnels. On note que les proximités organisées ou les proximités géographiques évoluent en fonction des problèmes auxquels doit faire face le territoire (Torre et Beuret, 2012).

Les recompositions territoriales décrites prennent à revers la logique de l'État et consacrent l'expression des initiatives locales. Elles sont la manifestation d'un besoin de plus de participation au niveau local et de prise en compte des valeurs endogènes dans les projets de territorialisation hydraulique. Cependant, on peut se demander si les schémas d'aménagement et de gestion des eaux en cours d'élaboration aideront à faire émerger clairement les intérêts des acteurs et à identifier leurs stratégies. Seront-ils à même de proposer des solutions de gestion plus intégratives agissant en faveur de la réduction du différentiel entre les proximités existantes et les proximités attendues, donc de mise en œuvre d'une dynamique de construction territoriale.

\section{Conclusion}

48 À partir d'une approche analytique se référant au concept de proximité, cet article contribue à expliquer l'inertie des CLE mis en place dans le cadre de la politique de la GIRE au Burkina Faso. Le fonctionnement des CLE pose la question des interactions sociospatiales entre les acteurs, des modalités de coordination et des dynamiques de la gestion de l'eau. À ce titre, l'approche en termes de proximité permet de rendre compte de l'articulation complexe entre les processus de construction des territoires, et les effets de contiguïté et de voisinage entre les usagers de l'eau.

Nos résultats montrent que les difficultés des CLE proviennent de la mise en proximité elle-même, qui se révèle asymétrique tant dans sa composante géographique qu'organisée. Les interdépendances vis-à-vis des ressources en eau dans les sous-bassins sont source de problèmes de gestion liés à la position spatiale des acteurs et aux relations passées ayant entaché le partage de l'eau. Ainsi, la création d'une structure ad hoc, le CLE, ne doit pas présager a priori de l'efficacité de la coordination. Si le CLE a permis l'activation de nouvelles interactions formalisées de proximité, cela n'a pas suffi à insuffler une véritable dynamique de gestion concertée des interdépendances autour de l'eau. Le CLE est loin de remettre en cause les logiques et rapports de force antérieurs entre les acteurs. Il témoigne surtout d'une inadaptation des politiques territoriales à des espaces institutionnels donnés et des territoires dont les limites se déplacent sous l'influence du jeu des proximités. Dans ce sens, l'exemple des CLE parait représentatif des difficultés et des conditions de réussite possible d'une territorialisation des politiques de l'eau.

50 Au niveau théorique, l'analyse des CLE montre la plasticité des relations de proximité, et surtout leur caractère dynamique. 


\section{BIBLIOGRAPHIE}

Barnèche-Miqueu L., Lahaye N., 2005, « Des réseaux d'acteurs locaux pour des projets de territoire. Les cas des secteurs électrique et métallurgique pyrénéens », in Guillaume R., Globalisation, systèmes productifs et dynamiques territoriales, Paris, L'Harmattan, p. 115-148.

Baron C., Bonnassieux A., 2013, « Gouvernance hybride, participation et accès à l'eau potable : le cas associations d'usagers de l'eau (AUE) au Burkina Faso ", Annales de géographie, $\mathrm{n}^{\circ}$ 693, p. 525-548.

BEGE (Bureau d'étude des géosciences et de l'environnement) ; DGRE (Direction générale des ressources en eau), 2008, Mission d'évaluation des processus de mise en place des comités locaux de l'eau et de leur opérationnalité, Ouagadougou, DGRE.

Blondiaux L., Sintomer Y., 2009, « L'impératif délibératif », Rue Descartes nº 63, p. 28-38.

Carrincazeaux C., Lung Y., Vicente J., 2008, « The scientific trajectory of the french school of proximity: interaction and institution - based approaches to Regional System of innovation ", European Planning Studies, vol. 16, $\mathrm{n}^{\circ}$ 5, 617-628.

D’Aquino P., 2002, « Le territoire entre espace et pouvoir : pour une planification territoriale ascendante ", L'Espace géographique, t. 31, p. 3-23.

De Fraiture et al., 2014, « Pirates or pioneers ? Unplanned irrigation around small reservoirs in Burkina Faso », Agricultural Water Management, n 131, p. 212-220.

DGIRH (Direction générale de l'inventaire des ressources hydrauliques), 2004, Les Comités locaux de l'eau (CLE) - Maillons de base du cadre institutionnel de la Gestion intégrée des ressources en eau au Burkina Faso, Ouagadougou, gouvernement du Burkina Faso.

DGRE (Direction générale des ressources en eau), 2006, État de la mise en œuvre du plan d'action pour la gestion intégrée des ressources en eau (PAGIRE) du Burkina Faso, mars 2003-juin 2006, Ouagadougou, gouvernement du Burkina Faso.

DGRE (Direction générale des ressources en eau), 2010, Les Comités locaux de l'eau (CLE) maillons de base du cadre institutionnel de Gestion intégrée des ressources en eau du Burkina Faso, Ouagadougou, gouvernement du Burkina Faso.

Diallo M., Wellens J., Dakouré D., Compaoré N. F., 2006, «L'expérience du projet Gestion de l'eau (GEeau) dans le bassin du Kou au Burkina Faso : promouvoir des approches innovantes de recherche-développement », non publié, p. 1-11.

Gallego-Ayala J., 2013, « Trends in integrated water resources management research: A literature review », Water Policy n 15, p. 628-647. doi:10.2166/wp.2013.149.

Ghiotti S., 2006, « Les Territoires de l'eau et la décentralisation. La gouvernance de bassin ou les limites d'une évidence », Développement durable et territoire, dossier 6, http://

developpementdurable.revues.org/document1742.html.

Gilly J., Torre, A., 2000, « Dynamiques de proximité : introduction », in Gilly J., Torre A., Dynamiques de proximité, Paris, L'Harmattan, p. 9-33.

Global Water Partnership (GWP), 2000, Integrated Water Resources Management, Stockholm, TAC Background Papers No. 4, Global Water Partnership. 
Gomez P. Y., Rousseau A., Vandangeon-Derumez, I., 2011, « Distance et proximité », Revue française de gestion, $\mathrm{n}^{\circ} 213$, p. 13-23.

Grigg N. S., 2008, «Integrated water resources management: Balancing views and improving practice », Water International, $\mathrm{n}^{\circ}$ 33, p. 279-292. doi: 10.1080/02508060802272820.

Leloup F., Moyart L., Pecqueur, B., 2005, « La gouvernance territoriale comme nouveau mode de coordination territoriale », vol. 7, Géographie Économie Société, p 321-332.

Mehta L., et al., 2014, « The politics of IWRM in Southern Africa », International Journal of Water Resources Development, $\mathrm{n}^{\circ}$ 30, p. 528-542. doi:10.1080/07900627.2014.916200.

Palé F. K., 2012, « Genèse et évolution d'un grand aménagement hydro-agricole : le périmètre rizicole de la vallée du Kou (Burkina Faso) » АНOHO, n 9, p. 1-13.

Pecqueur B., Zimmermann, J. B., 2004, «Introduction », in Pecqueur B., Zimmermann J. (dir) Économie de Proximités, Paris, Hermès Science Publication Lavoisier, p. 13-41

Rallet A., Torre, A., 1995, Économie industrielle - Économie spatiale, Paris, Economica.

RERU (Revue d'économie régionale et urbaine), 1993, « Économie de proximités », RERU, vol. 3, n ○ spécial.

Romagny B., Riaux J., 2009, « La gestion communautaire de l'eau agricole à l'épreuve des politiques participatives : regards croisés Tunisie/Maroc », Hydrological Sciences Journal, p. $1179-1196$.

Roncoli C. et al., 2009, « From management to negotiation: Technical and institutional innovations for integrated water resource management in the Upper Comoé River Basin, Burkina Faso ", Environmental Management, vol. 44, nº 4, p. 695-711.

Sally H., Lévite H., Cour J., 2011, « Local Water Management of Small Reservoirs: Lessons from Two Case Studies in Burkina Faso », Water Alternatives, vol. 4, n 3, p. 365-382.

Talbot D., 2010, «La dimension politique dans l'approche de la proximité », Géographie, économie, société, vol. $12, \mathrm{n}^{\circ} 2,1-26$.

Torre A., 2009, « Retour sur la notion de proximité géographique », Géographie, Économie, Société, vol. $11, \mathrm{n}^{\circ} 1,63-75$.

Torre A., Beuret, J.-E., 2012, Proximités territoriales. Construire la gouvernance des territoires, entre conventions, conflits et concertations, Paris, Economica-Anthropos.

Torre A., Zuindeau B., 2009, « Les apports de l'économie de la proximité aux approches environnementales : inventaire et perspectives », Natures Sciences, Dossier « Économie de la proximité », vol. 17, p. 349-360.

Venot J.-P., Torou B. M., Daré W., 2014, « Territorialisation ou spatialisation : les agences et comités locaux de l'eau au Burkina Faso », Espace géographique, n² 2, p. 148-163.

Warner J. F., 2006, « More sustainable participation? Multi-stakeholder platforms for integrated catchment management », International Journal of Water Resources Development, vol. 22, $\mathrm{n}^{\circ} 1$, p. 15-35.

Warner J., Simpungwe, E., 2012, « La participation des usagers dans la gestion de l'eau : quelles perspectives pour les plateformes multi-acteurs en Afrique subsaharienne ? », in Julien F. (dir), La gestion intégrée des ressources en eau en Afrique subsaharienne; Paradigme occidental, pratiques africaines, Québec, Presses de l'université de Québec, coll. » Géographie contemporaine », p. 107-133. 
Warner J., Wester P., Bolding A., 2008, « Going with the flow: river basins as the natural units for water management?», Water Policy , vol. 10, supplement, n² 2, p. 121-138.

Wellens J., Compaoré, N.-F., 2005, « Renforcement structurel de la capacité de gestion des ressources en eau pour l'irrigation dans le bassin du Kou », colloque « Salon africain de l'irrigation et du drainage », 6 décembre 2005, Ouagadougou.

Zoungrana T. P., 2010, « Les recompositions territoriales dans les aires des aménagements hydroagricoles : une riposte des acteurs locaux face à la logique verticale de l'État », in Igué J. O., Logique paysanne et espace agraire en Afrique, Paris, Karthala, vol. 5, p. 201-14.

\section{NOTES}

1. Voir Molle (2012).

2. La loi nº 002-2001/AN du 08 février 2001 portant loi d'orientation relative à la gestion de l'eau.

3. Challenge Program for Water and Food (CPWF) Volta - Composante 4. http:// volta.waterandfood.org/.

4. Le bureau est l'organe exécutif du CLE.

5. La fermeture des vannes de la prise d'eau a provoqué une montée des eaux qui ont débordé sur le village.

6. Entretien avec le directeur régional de l'ONEA.

7. Création par arrêté d'une autorité administrative territoriale.

8. Entretien avec les irrigants.

\section{RÉSUMÉS}

S'inscrivant dans une logique de compréhension des dynamiques de territorialisation, notre article porte sur l'analyse des modes de coordination entre acteurs locaux en vue d'une gestion intégrée des ressources en eau au Burkina Faso. En mobilisant le cadre d'analyse de la proximité, nous montrons que les dynamiques de relations socio-spatiales autour de la ressource en eau sont asymétriques et que la prise en compte (ou non) de cette inégalité spatiale peut favoriser ou, au contraire, nuire à la pérennité de la coordination. L'approche en termes de proximité permet de rendre compte de l'articulation complexe entre les processus de construction des territoires, et les principes d'intégration et de subsidiarité présentés comme gages de succès de la GIRE.

Our paper aims to analyze the modalities of coordination among local actors who are involved in integrated water resources management. So as to understand the dynamics of territorialization, we ground our analysis in the proximity framework to highlight that social and spatial relations around water resources are unbalanced. How this spatial inequality is taken into account may favor, or on the contrary, harm the sustainability of coordination. The approach in terms of proximity allows us to account for the complex articulation between the processes of territorial construction and the subsidiarity and participation principles presented as gage of IWRM success. 
INDEX

Mots-clés : gestion des ressources en eau, Comités locaux de l'eau (CLE), territoire, proximité, inégalités spatiales, conflit, Burkina Faso

Keywords : water resources management, local Water Committee, territory, proximity, spatial inequalities, conflict, Burkina Faso

\section{AUTEURS}

\section{BIO MOHAMADOU TOROU}

Bio Mohamadou Torou est assistant de recherche à l'International Water Management Institute et doctorant en géographie, université de Ouagadougou ; thème : « Pour une gestion intégrée des ressources en eau au Burkina Faso : cas des sous-bassins du Kou et de Yitenga ».

btorou@gmail.com

\section{LIZA DEBEVEC}

Liza Debevec est anthropologue à l'IWMI. Outre les questions liées à l'eau, ses recherches portent sur les aspects anthropologiques des pratiques alimentaires, le sexe, la vie quotidienne et la religion.1.debevec@cgiar.org

\section{DAPOLA ÉVARISTE CONSTANT DA}

Dapola Évariste Constant Da est professeur titulaire de géographie, HDR à l'université de Ouagadougou, actuellement, responsable de l'équipe de recherche Gestion des ressources naturelles du Laboratoire dynamique des espaces et sociétés (LDES). evaristeda@gmail.com 